\title{
Preparação de Argila Modificada com Cloreto de Cetilpiridíneo e Avaliação da Interação desta com o PVC
}

\author{
Daniel K. Resende, Camila B. Dornelas, Maria I. B. Tavares, Ailton S. Gomes, Leonardo A. Moreira \\ Instituto de Macromoléculas, UFRJ \\ Laboratório de Tecnologia Industrial Farmacêutica, UFRJ \\ Luiz A. Simeoni \\ Laboratório de Farmacologia Molecular, UnB
}

\begin{abstract}
Resumo: Foi preparada uma argila modificada com cloreto de cetilpiridíneo a partir da argila sódica por troca de cátions em solução. Foi avaliada a quantidade de agente de modificação em relação à argila sódica e o tempo reacional. Os materiais obtidos foram caracterizados por difração de raio X (XRD), análise termogravimétrica (TGA) e ressonância magnética nuclear (RMN) de baixo campo. Após a caracterização foi confirmada a modificação da argila e, também, foi verificado que o produto obtido pode ser empregado na preparação de nanocompósitos de PVC, considerando que o início da degradação do material preparado ocorreu em temperatura superior às comumente utilizadas no processamento do polímero. A adição da argila modificada apresentou uma dispersão adequada no PVC e manteve o início da degradação do material em temperatura compatível com o processamento do polímero, gerando um nanocompósito com parte esfoliada e intercalada.
\end{abstract}

Palavras-chave: Montmorilonita, PVC, XRD, RMN de baixo campo.

\section{Preparation of Modified Clay with Cetylpyridinium Chloride and Evaluation of Their Interaction with PVC}

Abstract: A modified silicate with cetylpyridinium was prepared from sodium clay with cation exchange in solution. The amount of modification agent for clay and the reaction time were evaluated. The materials produced were characterized using X ray diffraction (XRD), termogravimetric analysis (TGA) and low field nuclear magnetic resonance (NMR). The formation of new organic clay was confirmed, which was introduced in PVC for the formation of nanocomposites. The beginning of degradation of the new clay occurred at temperatures higher than commonly used in the processing of PVC. The nanocomposites were partially exfoliated and partially intercalated.

Keywords: Montmorillonite, PVC, XRD, low field NMR.

\section{Introdução}

O poli(cloreto de vinila) (PVC) é um dos termoplásticos mais consumidos no mundo, sendo considerado o mais versátil dentre os plásticos. A grande versatilidade do PVC se deve as suas propriedades e também a sua adequação aos mais variados processos de transformação. Uma vez que a resina é atóxica e inerte, a escolha de aditivos com essas mesmas características permite a fabricação de filmes para embalagens de alimentos e farmacêuticas, além de produtos médico-hospitalares ${ }^{[1,2]}$.

Uma característica que limita muito o processamento do PVC é o fato desse polímero ser menos estável que muitos materiais plásticos, sofrendo um processo de degradação devido ao calor, oxigênio, luz e energia mecânica ${ }^{[3]}$. Pode-se, também, preparar compósitos com outros polímeros para se melhorar e/ou modificar as propriedades mecânicas e térmicas do PVC, como blendas com o polietileno ${ }^{[3]}$ e policloropreno ${ }^{[4]}$. Os compósitos podem ser definidos como materiais que contém duas ou mais substâncias combinadas, produzindo um material com propriedades funcionais e estruturais diferentes daquelas dos constituintes individuais. São heterogêneos e multifásicos, sendo um componente descontínuo (estrutural ou de reforço) que fornece resistência ao material, e outro, que representa o meio de transferência desse esforço (matricial ou fase contínua). Quando o componente descontínuo está disperso em escala nanométrica, o termo utilizado para o material produzido é nanocompósito ${ }^{[5]}$. Silicatos lamelares, tanto naturais quanto sintetizados, têm sido muito usados na síntese de nanocompósitos poliméricos. As argilas do tipo montmorilonita, mica, hectorita e saponita são as mais comumente utilizadas como material de enchimento de polímeros devido às suas características únicas de intercalação e esfoliação ${ }^{[5]}$. Geralmente, o uso de materiais inorgânicos não apresenta uma interação boa com polímeros orgânicos pela falta de uma dispersão e adesão adequada. As modificações de superfície são comumente utilizadas para obter uma melhor interação da superfície da argila com a da matriz polimérica. As trocas dos íons de sódio e cálcio presentes nas lamelas das argilas por íons alquilamônio é uma das alternativas utilizadas para levar à modificação da característica hidrofílica entre as lamelas para hidrofóbica, reduzindo também as forças físicas e eletrostáticas interlamelares, facilitando o processo de formação de nanocompósitos, bem como o processo de esfoliação ${ }^{[6]}$. A preparação de nanocompósitos usando argilas modificadas com octadecilamina ou montmorilonita sódica em polímeros como PVC têm como objetivo melhorar suas propriedades mecânicas e de barreira a gases do mesmo. Entretanto, essas argilas não têm apresentado boas propriedades referentes à estabilidade térmica ou dispersão aceitável da argila na matriz polimérica. As gerações de nanocompósitos de PVC usando argila comercial

Autor para correspondência: Maria I. B. Tavares, Centro de Tecnologia, Instituto de Macromoléculas, Universidade Federal do Rio de Janeiro - UFRJ, Av. Horácio Macedo, 2030, Bloco J, Sala J 207, Cidade Universitária, Ilha do Fundão, CEP: 21941-598, Rio de Janeiro, RJ, Brasil. E-mail: mibt@ima.ufrj.br 
sódica ou modificada com octadecilamina, visando aumentar as propriedades mecânicas e de barreira a gases, não apresentaram muito sucesso, porque essas argilas não apresentam estabilidade térmica ou dispersão adequada na matriz polimérica ${ }^{[7,8]}$. Assim, de acordo com o exposto, o objetivo desse trabalho foi preparar uma argila modificada com cloreto de cetilpiridíneo, para obter uma argila organofílica com melhor estabilidade térmica e a avaliação da adição desta na formação de nanocompósitos com o PVC via intercalação por solução.

\section{Experimental}

\section{Preparo da argila modificada}

A técnica de modificação da argila consistiu na dispersão da argila sódica no solvente de escolha, no caso, a água, e posterior adição do surfactante cloreto de cetilpiridíneo solubilizado, mantendo o sistema sob agitação. Nos ensaios realizados, avaliou-se o efeito da variação da relação argila sódica:surfactante e do tempo reacional. A argila sódica utilizada nos experimentos apresentava uma capacidade de troca catiônica (CEC) de 90 mmoles/100 g. As argilas modificadas foram preparadas com a proporção aproximada de 6:1, 3:1 e 2:1 (p/p) de argila sódica:surfactante. Na condição de 6:1 foi adicionada uma quantidade de surfactante próxima da metade da CEC, na segunda condição foi adicionado um excesso de $20 \%$ de surfactante a mais do que o necessário para atingir a capacidade de troca catiônica e na última foi empregado um excesso de $80 \%$ de surfactante em relação a CEC da argila sódica. As reações foram realizadas sob temperatura ambiente, variando-se o tempo de reação de 1 até 24 horas de reação. O produto das reações foi levado à filtração. O precipitado foi seco em dessecador durante alguns dias e, então, pulverizado.

\section{Preparação das membranas de PVC}

O polímero PVC foi adicionado lentamente em tetrahidrofurano (THF) e deixado sob agitação até a completa dissolução do polímero no solvente. Foi adicionada a argila modificada com o cetilpiridíneo na proporção de 97:3 polímero:argila no frasco e mantido o meio sob agitação por 24 horas à temperatura ambiente. Após esse período o material foi retirado do frasco, vertendo o líquido sobre placas de Petri, mantendo as mesmas a temperatura ambiente em uma capela até a evaporação do solvente e formação das membranas. Foram preparadas membranas de PVC contendo a argila modificada com o cetilpiridíneo e uma segunda argila comercial (Viscogel B8), que já é modificada com octadecilamina, com a finalidade de se comparar o efeito que as mesmas iriam apresentar quando da interação com o polímero. Os materiais obtidos foram caracterizados.

\section{Caracterização}

As modificações efetuadas nas argilas podem ser detectáveis por difração de raio $\mathrm{X}$, conforme verificado em estudos anteriores ${ }^{[9,10]}$. Uma nova técnica que pode ser usada é o RMN de baixo campo ${ }^{[11]}$, porque permite a observação da formação de nanocompósitos pelo aparecimento de domínios de diferentes mobilidades moleculares.

A avaliação da estabilidade térmica dos materiais modificados por ser verificada através de análise termogravimétrica.

\section{Difração de raio $X$}

As amostras dos nanomateriais preparados, obtidos a partir da modificação da argila sódica, foram pulverizadas e analisadas em um difratômetro de raio $\mathrm{X}$ operado a $30 \mathrm{kV}, 15 \mathrm{~mA}$, com um passo de 0,05 e taxa de varredura de $1{ }^{\circ} \mathrm{C} / \mathrm{min}$ na temperatura ambiente. A radiação $\mathrm{CuK}_{\alpha}$ foi utilizada como fonte dos raio $\mathrm{X}$, com comprimento de onda de $0,15418 \mathrm{~nm}$. A faixa de varredura da difração foi ajustada para ângulos de 2 a $20^{\circ}$. Além das amostras reacionais, foi preparada também a amostra pura da argila sódica para avaliação do espaçamento basal inicial entre as lamelas da argila.

\section{RMN de baixo campo}

Quando os spins nucleares são excitados e essa frequência de ressonância é retirada os núcleos voltam ao estado fundamental emitindo energia na região de radiofrequência, sendo o processo de retorno ao equilíbrio denominado de processo de relaxação. Ocorrem dois processos de relaxação dos núcleos em paralelo. Um deles é entálpico e emite energia térmica para a rede, sendo assim denominado de spin-rede ou longitudinal, com constante de tempo $\mathrm{T}_{1}$. O outro processo é entrópico e é denominado spin-spin, tendo como constante de tempo $\mathrm{T}_{2}$. Neste trabalho foi determinado o tempo de relaxação $T_{1}$ para o núcleo de hidrogênio $\left(T_{1} H\right)$, pois este processo fornece informações importantes sobre a dinâmica molecular da amostra, em face das modificações sofridas pelo material. Essa informação é registrada pelo modo com que os núcleos relaxam. Neste processo o excesso de energia a ser emitida para a rede é doado sob a forma de interação dipolar, ocorrendo um abaixamento da entalpia do sistema de spins nucleares. $\mathrm{O}$ retorno da magnetização é usualmente exponencial, sendo, portanto um processo de primeira ordem.

Para a determinação das medidas de relaxação nuclear foi utilizado um espectrômetro de RMN de baixo campo Maran Ultra 23, operando a frequencia de $23 \mathrm{MHz}$ (para o núcleo de hidrogênio) e equipado com sonda de $18 \mathrm{~mm}$ com variação de temperatura. O tempo de relaxação spin-rede do hidrogênio foi determinado diretamente pela sequencia de pulso inversão-recuperação $\left(180^{\circ}-\tau-90^{\circ}\right)$, o pulso de 90 foi calibrado automaticamente pelo software do equipamento. A amplitude do FID foi de 20 pontos variando de 0,1 a $5000 \mathrm{~ms}$, com 4 medidas para cada ponto com um intervalo de reciclo de 5 segundos. Os valores e as intensidades relativas de relaxação foram obtidas pelo fiting dos dados exponenciais com a ajuda do Programa do equipamento denominado WINFIT.

\section{Análise termogravimétrica}

As amostras foram colocadas em um suporte de alumínio presente no equipamento e analisadas em fluxo de nitrogênio com vazão de $50 \mathrm{~mL} / \mathrm{min}$, com uma taxa de aquecimento de $20^{\circ} \mathrm{C} / \mathrm{min}$ de 50 a $750{ }^{\circ} \mathrm{C}$.

\section{Resultados e Discussão}

\section{Caracterização da argila modificada com cetilpiridíneo}

\section{Análise de difração de raio X}

Nas Figuras 1, 2 e 3 estão apresentados as reações de modificação de argila com quantidade diferentes de surfactantes em relação a argila sódica. Quando utilizada a proporção maior de argila sódica (6:1), verificou-se que a quantidade foi insuficiente para promover a intercalação da argila, sendo isso verificada pela menor expansão do espaçamento basal. Para a proporção intermediária de argila sódica:surfactante (3:1) verificou-se que esta é a quantidade de surfactante para a reação de troca na argila, sendo observado um maior espaçamento basal para a argila modificada em relação a argila sódica (expansão de $13 \AA$ para valores próximos a $22 \AA$ ), quando comparada com a proporção de $6: 1$. Na proporção de 2:1 verificou-se a manutenção do espaçamento basal verificado na proporção de 3:1, entretanto nos tempos reacionais de 1 e 2 horas observou-se também o aparecimento de um segundo sinal na difração 
de raio X com espaçamento basal próximo a 39 Å (Tabela 1). Esse aparecimento do segundo sinal parece estar relacionado à formação de bicamadas como já verificadas em reações na literatura em que são empregadas quantidades excessivas de surfactante ${ }^{[12]}$.

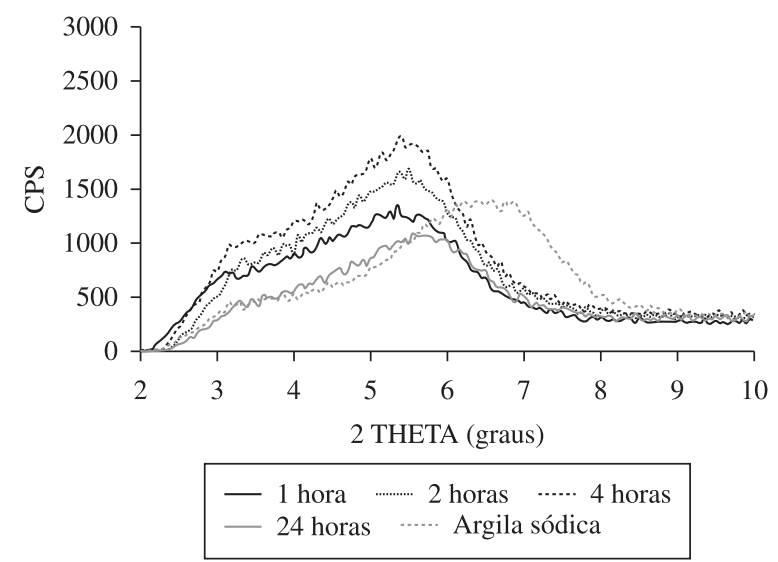

Figura 1. Modificação da argila sódica:cetilpiridíneo na proporção 6:1.

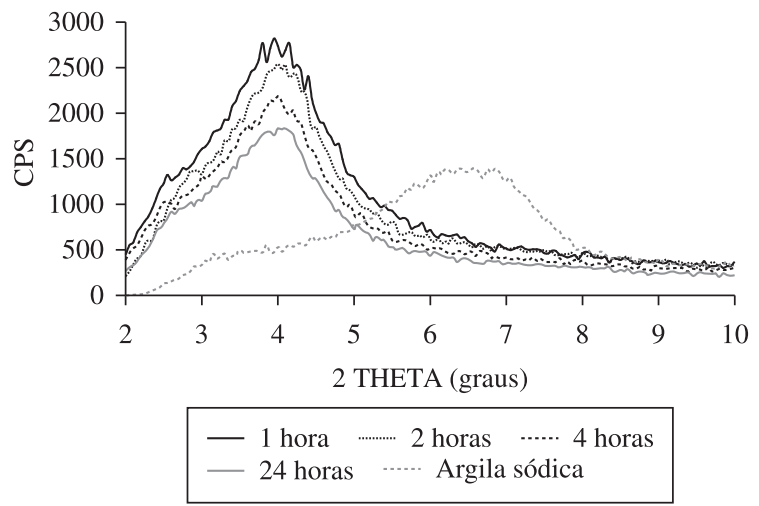

Figura 2. Modificação da argila sódica:cetilpiridíneo na proporção 3:1.

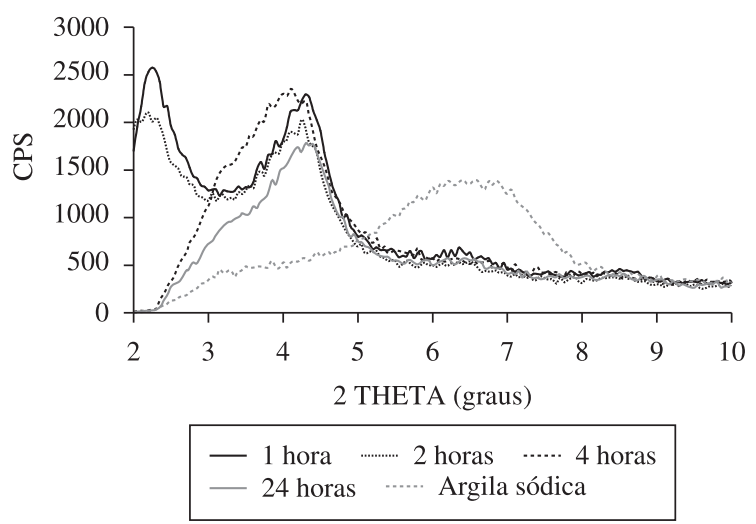

Figura 3. Modificação da argila sódica:cetilpiridíneo na proporção 2:1.

Tabela 1. Resultados da difração de raio X com concentrações distintas de cetilpiridíneo no meio reacional.

\begin{tabular}{ccc}
\hline $\begin{array}{c}\text { Proporção de argila sódica: } \\
\text { surfactante }(\mathbf{p} / \mathbf{p}) \text {, tempo } \\
\text { reacional }\end{array}$ & $\mathbf{2}$ Theta $(\theta)$ & $\begin{array}{c}\text { Espaçamento } \\
\text { basal }(\mathbf{\AA})\end{array}$ \\
\hline Argila sódica & 6,55 & 13,5 \\
$6: 1,1$ hora & 5,35 & 16,5 \\
$3: 1,1$ hora & 3,95 & 22,2 \\
$2: 1,1$ hora & $2,25 / 3,95$ & $39,3 / 22,2$ \\
\hline
\end{tabular}

Considerando que aminas alifáticas intercaladas em argila apresentaram em estudos anteriores efeitos deletérios na preparação de materiais com PVC quando empregadas em maiores quantidades ${ }^{[7,8]}$, optou-se por se trabalhar com a argila obtida pela reação com a proporção de $3: 1$. Ao se avaliar os tempos reacionais das reações na proporção de 3:1 foram observados tempos satisfatórios para obtenção de intercalação do cloreto de cetilpiridíneo na argila já em 1, 2 e 4 horas de reação (Figura 2). Entretanto como se observou já um valor de espaço basal levemente superior para o período de 1 hora de tempo reacional, optou-se em continuar a avaliação com tempos reacionais de 1 e 2 horas. Como não foram verificadas variações no espaçamento basal que justificassem os tempos reacionais de 4 e 24 horas, os mesmos foram excluídos de análises futuras.

\section{Análises termogravimétricas}

Nas Figuras 4 e 5 estão apresentadas as reações preparadas com a proporção de 3:1 argila sódica:surfactante nos tempos reacionais de 1 e 2 horas, respectivamente. Foi verificado que no tempo reacional de 1 hora o valor de onset foi um pouco superior ao verificado em 2 horas de tempo reacional, embora no tempo reacional de 2 horas tenha ocorrido um percentual de intercalação $3 \%$ superior de na argila (Tabela 2).

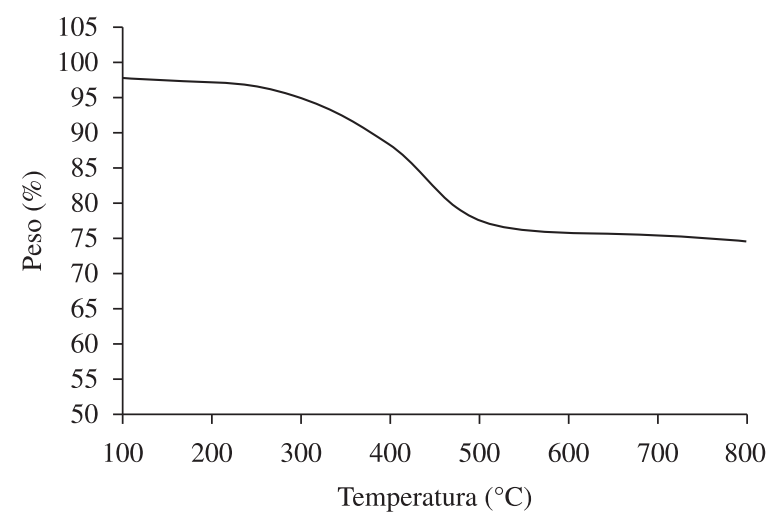

Figura 4. TGA da argila modificada com cloreto de cetilpiridíneo (tempo reacional 1 hora).

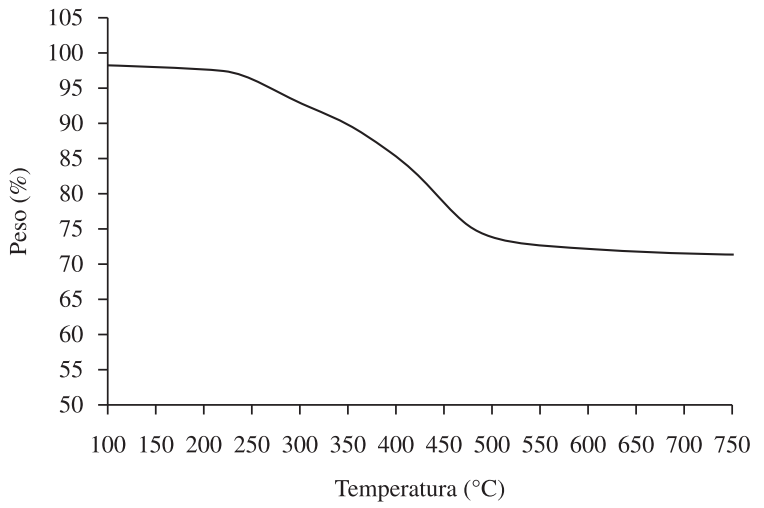

Figura 5. TGA da argila modificada com cloreto de cetilpiridíneo (tempo reacional 2 horas)

Tabela 2. Análise de TGA das argilas modificadas com cetilpiridíneo.

\begin{tabular}{ccc}
\hline $\begin{array}{c}\text { Argila modificada - } \\
\text { proporção 3:1 }(\mathbf{p} / \mathbf{p}) \text { tempo } \\
\text { reacional }\end{array}$ & $\begin{array}{c}\text { Perda de massa } \\
\left(\mathbf{1 5 0 - 6 5 0}{ }^{\circ} \mathbf{C}\right)\end{array}$ & $\begin{array}{c}\text { Temperatura } \\
\text { de onset }\left({ }^{\circ} \mathbf{C}\right)\end{array}$ \\
\hline 1 hora & $22,6 \%$ & 351 \\
2 horas & $25,9 \%$ & 318 \\
\hline
\end{tabular}


Tabela 3. Valores de $\mathrm{T}_{1} \mathrm{H}$ das amostras avaliadas no RMN de baixo campo para as matérias-primas.

\begin{tabular}{|c|c|c|c|c|c|}
\hline \multirow{2}{*}{$\begin{array}{l}\text { Matérias primas } \\
\text { Argila sódica }\end{array}$} & \multicolumn{4}{|c|}{$\begin{array}{c}\mathrm{T}_{1} \mathrm{H}(\mathrm{ms}) \text { e porcentagem }(\%) \text { dos domínios nas matérias-primas utilizadas } \\
\text { como materiais de partida na formação das argilas modificadas }\end{array}$} & \multirow{2}{*}{$\begin{array}{c}\text { No. de domínios } \\
3\end{array}$} \\
\hline & $0,01(10,5 \%)$ & $0,50(88,9 \%)$ & $14,4(0,5 \%)$ & & \\
\hline & $0,01(7,3 \%)$ & $0,50(90,6 \%)$ & $6,70(2,1 \%)$ & & 3 \\
\hline & $0,01(7,5 \%)$ & $0,50(92,5 \%)$ & $* *$ & & 2 \\
\hline \multirow[t]{3}{*}{ CCP* } & $0,01(41,8 \%)$ & $2,10(0,7 \%)$ & $72,9(1,8 \%)$ & $5535,6(55.7 \%)$ & 4 \\
\hline & $0,01(41,6 \%)$ & $2,60(0,7 \%)$ & $47,2(1.7 \%)$ & $5541,7(56.0 \%)$ & 4 \\
\hline & $0,01(41.7 \%)$ & $4,30(0,8 \%)$ & $84,2(2.0 \%)$ & $5882,1(55,5 \%)$ & 4 \\
\hline
\end{tabular}

* O agente de modificação da argila sódica foi o Cloreto de cetilpiridíneo (CCP). ** Não foi observado o respectivo domínio na análise de baixo campo.

Tabela 4. Valores de $\mathrm{T}_{1} \mathrm{H}$ das amostras avaliadas no RMN de baixo campo para a argila modificada.

\begin{tabular}{cccc}
\hline $\begin{array}{c}\text { Argila modificada } \\
\text { (tempo reacional) }\end{array}$ & $\begin{array}{c}\mathbf{T}_{1} \mathrm{H}(\mathbf{m s}) \text { e porcentagem (\%) } \\
\text { dos domínios }\end{array}$ & $\begin{array}{c}\text { No. de } \\
\text { domínios }\end{array}$ \\
\hline CCP (1 hora) & $0,60(100 \%)$ & $*$ & 1 \\
& $0,60(100 \%)$ & $*$ & 1 \\
& $0,60(100 \%)$ & $*$ & 1 \\
CCP (2 horas) & $0,50(96,5 \%)$ & $43,4(3,5 \%)$ & 2 \\
& $0,60(96,9 \%)$ & $25,2(3,1 \%)$ & 2 \\
& $0,50(93,8 \%)$ & $11,7(6,2 \%)$ & 2 \\
\hline
\end{tabular}

*Não foi observado o respectivo domínio na análise de baixo campo.

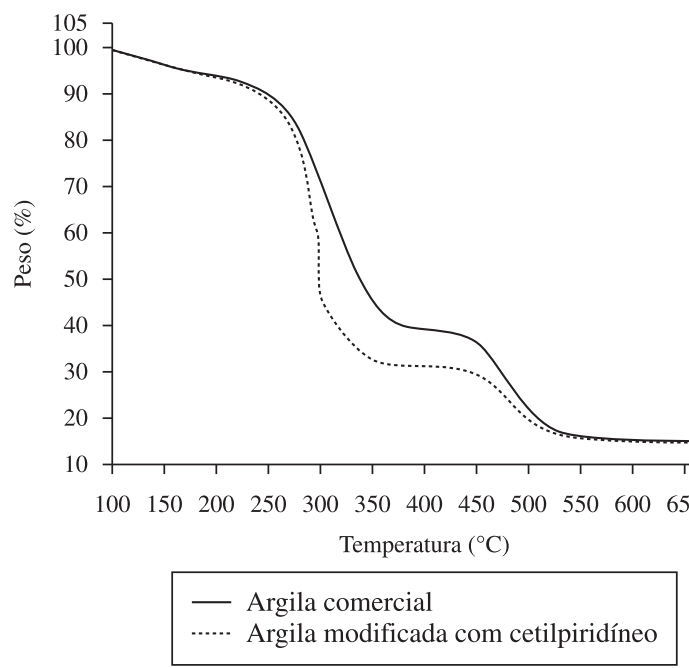

Figura 6. Análise de TGA da membrana de PVC com a argila comercial e da modificada com cetilpiridíneo.

Foram observados no TGA temperaturas de onset superiores à temperatura de processamento do $\mathrm{PVC}\left(=<180{ }^{\circ} \mathrm{C}\right)^{[7,8]}$ para a argila modificada (Figura 4 e 5). As argilas modificadas com cetilpiridíneo mostraram-se estáveis.

\section{Análises de RMN de baixo campo}

Os dados de RMN de baixo campo obtidos para as matérias-primas utilizadas estão apresentados na Tabela 3 e os dos produtos preparados nas reações utilizando a proporção de 3:1 de argila sódica:surfactante constam na Tabela 4. Os ensaios foram feitos em triplicatas e os resultados desses ensaios estão apresentados nas Tabelas 3 e 4.

Nos resultados observados nos ensaios de RMN de baixo campo não foi diferenciado o domínio em $0,50 \mathrm{~ms}$ da argila sódica do formado após a modificação da argila. Como o processo utilizado para modificação foi o de intercalação em solução, seguido de filtração do material formado e, uma vez que foram utilizados agentes de modificação de baixo peso molecular, os domínios formados não se diferenciaram muito do já observado na argila sódica. No entanto, o RMN de baixo campo permitiu verificar a homogeneidade do material formado, visto a ausência de outros domínios e/ou uma predominância de um único domínio. Para o tempo reacional de 1 hora foi verificado um único domínio na argila modificada, o que assegura que os processos de preparação e separação do material modificado foram adequados.

\section{Avaliação das membranas de PVC}

Foram feitas análises térmicas das membranas de PVC e os resultados de TGA das mesmas estão apresentados na Figura 6.

Segundo o TGA, as temperaturas de onset observadas para os dois foram próximas a $270{ }^{\circ} \mathrm{C}$, contudo para a argila modificada com cetilpiridíneo foi verificada uma degradação mais acelerada do PVC após o onset.

\section{Conclusões}

Foi observado que com o agente de intercalação nos tempos reacionais de 1 e 2 horas já foram suficientes para intercalação do surfactante na argila com a difração de raio X. Essas argilas modificadas apresentaram temperaturas de onset superiores à de processamento do PVC, sendo verificada alta estabilidade para a argila modificada.

O RMN de baixo campo permitiu verificar parâmetros reacionais como: adequada solubilização do material antes do processo de intercalação, adequada remoção dos resíduos de material não intercalado por filtração e homogeneidade do nanomaterial formado, podendo ser considerada uma poderosa ferramenta para ajuste dos procedimentos operacionais a serem utilizados na modificação de argilas e preparação de nanomateriais.

As técnicas de XRD, TGA e RMN de baixo campo associadas permitiram a confirmação da formação das argilas modificadas e a homogeneidade das mesmas.

A degradação térmica do material obtido do PVC com a argila modificada iniciou em temperatura superior a $200{ }^{\circ} \mathrm{C}$. Se for considerado que as temperaturas normalmente utilizadas no processamento do PVC estão entre 140 até $180^{\circ} \mathrm{C}$, esses resultados podem indicar a possibilidade do uso dessa argila organofílica para preparação de nanomateriais com PVC. Na comparação com a argila comercial não foi verificado aumento nas temperaturas em que se iniciava o processo de degradação do polímero, mas caberia, ainda, avaliação de outras condições reacionais e análises mecânicas para se avaliar se a argila modificada com cetilpiridíneo poderia ou não gerar incremento de outras propriedades da membrana de PVC.

\section{Agradecimentos}

Ao suporte provido pelo CNPq e CAPES.

\section{Referências Bibliográficas}

1. Vinhas, G. M.; Souto-Maior, R. M. \& Almeida, Y. M. B. - Polímeros, 15, p.207-215 (2005)

2. Henz, G. P. \& Reifschneider, F. J. B. - Horticultura Brasileira, 23, p.61-67 (2005).

3. Vrandecic, N. S.; Andricic, B.; Klaric, I. \& Kovacic, T. - Polymer Degradation and Stability, 90, p.455-460 (2005). 
4. Saha, S. - European Polymer Journal, 37, p.399-410 (2001).

5. Mano, E. B. \& Mendes, L. C. - Avaliação das propriedades dos polímeros, editora Edgard Blucher, SP, Brazil, (1999).

6. Carrado, K. A. - "Applied Clay Science”, 17, p.1-23 (2000).

7. Wan, C.; Zhang, Y. \& Zhang, Y. - "Polymer Testing", 23, p.299-306 (2004).

8. Gong, F.; Feng, M.; Zhao, C.; Zhang, S. \& Yang, M. - "Polymer Degradation and Stability", 84, p.289-294 (2004).

9. Dornelas, C. B.; Resende, D. K.; Rocha, H. V. A.; Gomes, A. S., Tavares, M. I. B. - "Polímeros", 18, p.222-229 (2008).
10. Dornelas, C. B.; Resende, D. K.; Tavares, M. I. B. \& Gomes, A. S. "Polímeros", 18, p.187-192 (2008).

11. "Tavares, M. I. B.; Nogueira, R. F.; Gil, R.A.S.; Preto, M. et al. "Polymer Testing", 26, p.1100 -1102 (2007).

12. LeBaron, P. C.; Wang, Z. \& Pinnavaia, T. J. - "Applied Clay Science", 15, p.11-29 (1999)

Enviado: 06/11/09

Reenviado: $26 / 03 / 10$

Aceito: $05 / 04 / 10$

DOI: 10.1590/S0104-14282010005000031 